\title{
Color Image Reconstruction by Discrete Orthogonal Moment
}

\author{
J. S. Rivera-López, C. J. Camacho-Bello \\ Universidad Politécnica de Tulancingo, Tulancingo, México \\ Email:saul.rivera@upt.edu.mx,cesar.camacho@upt.edu.mx
}

How to cite this paper: Rivera-López, J.S. and Camacho-Bello, C.J. (2017) Color Image Reconstruction by Discrete Orthogonal Moment. Journal of Data Analysis and Information Processing, 5, 156-166. https://doi.org/10.4236/jdaip.2017.54012

Received: September 1, 2017

Accepted: November 14, 2017

Published: November 17, 2017

Copyright (c) 2017 by authors and Scientific Research Publishing Inc. This work is licensed under the Creative Commons Attribution International License (CC BY 4.0).

http://creativecommons.org/licenses/by/4.0/ C) (i) Open Access

\begin{abstract}
Image reconstruction can help to determine how well an image may be characterized by a small finite set of its moments. Also, we can identify the number of descriptors needed to describe an image. In this work, we present a comparative analysis using different set of discrete orthogonal moments in terms of normalized image reconstruction error (NIRE). Color image reconstruction is performed with different color channels and various orders of different discrete orthogonal moments. Finally the results obtained by the reconstruction of three color images with different families of orthogonal moments and an error analysis to compare their capacity of description are presented, also the conclusions obtained from this work are presented.
\end{abstract}

\section{Keywords}

Reconstructed Color Image, Discrete Orthogonal Moment, Comparative Analysis

\section{Introduction}

Nowadays, all digital cameras acquire chromatic images, which are capable of recording complex details. The conventional approach to color image analysis consists of processing the red, green and blue channels separately using digital image processing techniques, and combines the individual output results [1]. Currently, RGB images are analyzed with orthogonal quaternion moments, which have the advantage of using the quaternion theory to handle color image is that the existing correlation between color components can be taken into consideration [2]. B. Chen et al. proposed the quaternion moments for color images analysis [3]. The authors use the Zernike polynomials as the kernel of the quaternion moments defined in a unit circle. However, the computation of quaternion moments requires a transformation to polar coordinates and an approxi- 
mation of the integral [4]. The discrete orthogonal moments do not involve any numerical approximation, since the base set is orthogonal in the discrete domain of the image co-ordinate space, the basic functions satisfy exactly the orthogonality property, and thus yield a superior image reconstruction [5]. The classical discrete orthogonal moments such as Tchebichef [5], Krawtchouk [6], Hahn [7], Meixner [8], Charlier [9] and Racah [10] [11] moments are defined in the coordinate space of the image and preserve the orthogonality condition [7]. Has recently been analyzed color images with images reconstruction from the different channels by orthogonal moments such as Charlier [9] and Racah [10] moments.

In this article we present an analysis based on a reconstruction error of color images using different orders of moments, which allows knowing the capacity of reconstruction with different set of classical orthogonal moments is analyzed, also the recovery error graphs that were obtained are presented.

\section{Discrete Orthogonal Moments}

The discrete orthogonal moments have the ability to represent global features and describe its most important characteristics, which have a kernel base of discrete orthogonal polynomials [6].

The orthogonal moments are defined as follows:

$$
\varphi_{m, n}=\sum_{x=1}^{m} \sum_{y=1}^{n} f(x, y) P_{n}(x) P_{m}(y)
$$

where $P_{n}(x)$ and $P_{m}(x)$ are a set of discrete orthogonal $f(x, y)$ is an image function and $m, n$ are the image size.

Considering that a color image is formed by RGB channels they can be defined as,

$$
\begin{aligned}
& f(x, y, 1)=f_{R}(x, y), \\
& f(x, y, 2)=f_{G}(x, y), \\
& f(x, y, 3)=f_{B}(x, y),
\end{aligned}
$$

The calculation of the orthogonal moments is defined as

$$
\varphi_{n, m}^{c}=\sum_{x=1}^{m} \sum_{y=1}^{n} f(x, y, c) P_{n}(x) P_{m}(y)
$$

where $P_{n}(x)$ y $P_{m}(y)$ are polynomial basic functions, $f(x, y, c)$ is a color image, and $c$ represents the RGB channel of the image.

\section{Discrete Orthogonal Polynomials}

A general way to obtain normalized discrete orthogonal polynomials $\tilde{p}_{n}(x)$ is by the following recurrence relation (H. Zhu et al. [12]).

$$
A \tilde{p}_{n}(x)=B * D \tilde{p}_{n-1}(x)+C * E \tilde{p}_{n-2}(x)
$$

where A, $B, C, D, E$ are terms independent of each of the polynomial sets shown in "Table 1" and "Table 2" and $\tilde{p}_{n-1}(x), \tilde{p}_{n-2}(x)$ are the initial values in "Table 3" and "Table 4". 
Table 1. Values of the terms $A, B, C, D$ and $E$ of the following polynomial with size $N$ : Tchebichef $\tilde{t}_{n}(x ; N)$, Krawtchouk $\tilde{k}_{n}(x ; p, N)$ y Charlier $\tilde{c}_{n}^{a_{1}}(x)$.

\begin{tabular}{|c|c|c|c|}
\hline & $\tilde{t}_{n}(x ; N)$ & $\tilde{k}_{n}(x ; p, N)$ & $\tilde{c}_{n}^{a_{1}}(x)$ \\
\hline$A$ & $\frac{n}{2(2 n-1)}$ & $n$ & $-a_{1}$ \\
\hline$B$ & $x-\frac{N-1}{2}$ & $x-n+1-p(N-2 n+2)$ & $x-n+1-a_{1}$ \\
\hline$C$ & $-\frac{(n-1)\left(N^{2}-(n-1)^{2}\right)}{2(2 n-1)}$ & $-p(1-p)(N-n+2)$ & $n-1$ \\
\hline$D$ & $\sqrt{\frac{2 n+1}{\left(N^{2}-n^{2}\right)(n+1)}}$ & $\sqrt{\frac{n}{p(1-p)(N-n+1)}}$ & $\sqrt{\frac{a_{1}}{n}}$ \\
\hline$E$ & $\sqrt{\frac{2 n+1}{\left(N^{2}-n^{2}\right)\left(N^{2}-(n+1)\right)}} * \sqrt{\frac{1}{2 n-3}}$ & $\sqrt{\frac{n}{p(1-p)(N-n+2)}} * \sqrt{\frac{n-1}{N-n+1}}$ & $\sqrt{\frac{a_{1}^{2}}{n(n-1)}}$ \\
\hline
\end{tabular}

Table 2. Values of the terms $A, B, C, D$ and $E$ of the following polynomial with size $N: \operatorname{Hahn} \tilde{h}_{n}^{a, b}(x ; N)$, Meixner $\tilde{m}_{n}^{\beta, \mu}(x)$.

\begin{tabular}{|c|c|c|}
\hline & $\tilde{h}_{n}^{a, b}(x ; N)$ & $\tilde{m}_{n}^{\beta, \mu}(x)$ \\
\hline$A$ & $\frac{n}{a+b+2 n-1} * \frac{a+b+n}{a+b+2 n}$ & $\frac{\mu}{\mu-1}$ \\
\hline$B$ & $\begin{array}{l}x-\frac{a-b+2 N-2}{4} \\
-\frac{\left(b^{2}+a^{2}\right)(a+b+2 N)}{4(a+b+2 n-2)(a+b+2 n)}\end{array}$ & $\frac{x-x \mu-n+1-\mu n+\mu-\beta \mu}{1-\mu}$ \\
\hline$C$ & $\begin{array}{l}-\frac{(a+n-1)(N-n+2)}{a+b+2 n-1} \\
* \frac{(a+b+N+n-1)(N-n+1)}{a+b+2 n-1}\end{array}$ & $-\frac{(n-1)(n-2+\beta)}{1-\mu}$ \\
\hline$D$ & $\sqrt{\frac{n(a+b+n)(a+b+2 n+1)}{(a+n)(b+n)(a+b+n+N)(N-n)(a+b+2 n-1)}}$ & $\sqrt{\frac{\mu}{n(\beta+n-1)}}$ \\
\hline$E$ & $\begin{array}{l}\sqrt{\frac{n(n-1)(a+b+n)}{(a+n)(b+n)(a+n+1)(b+n+1)(N-n+1)(N-n)}} \\
* \sqrt{\frac{(a+b+n-1)(a+b+2 n)}{(a+b+2 n-3)(a+b+2 n-1)}}\end{array}$ & $\sqrt{\frac{\mu^{2}}{(n-1)(\beta+n-2) n(\beta+n-1)}}$ \\
\hline
\end{tabular}

A special case of the discrete orthogonal polynomials are the Racah polynomials, which are calculated by the following relation of recurrence with respect to $n[10][11]$.

$$
A_{n} U_{n}^{\alpha, \beta}(s, a, b)=B_{n} \frac{d_{n}}{d_{n-1}} U_{n-1}^{\alpha, \beta}(s, a, b) C_{n} \frac{d_{n}}{d_{n-2}} U_{n-2}^{\alpha, \beta}(s, a, b)
$$

where $A_{n}, B_{n}, C_{n}$ are terms independent of Racah polynomials and $U_{n-1}^{\alpha, \beta}(s, a, b)$, 
Table 3. Zero and first order polynomials of the following functions: Tchebichef $\tilde{t}_{n}(x ; N)$, Krawtchouk $\tilde{k}_{n}(x ; p, N)$ y Charlier $\tilde{C}_{n}^{a_{1}}(x)$.

\begin{tabular}{|c|c|c|c|}
\hline & $\tilde{t}_{n}(x ; N)$ & $\tilde{k}_{n}(x ; p, N)$ & $\tilde{c}_{n}^{a_{1}}(x)$ \\
\hline & 1 & $N ! p^{x}(1-p)^{N-x}$ & $\sqrt{\mathrm{e}^{-\mu} \mu^{x}}$ \\
\hline$p_{0}(x)$ & $\overline{\sqrt{N}}$ & $\sqrt{x !(N-x) !}$ & $\sqrt{\frac{\mu}{x !}}$ \\
\hline$\tilde{p}_{1}(x)$ & $(N-1-2 x) \sqrt{\frac{3}{N\left(N^{2}-1\right)}}$ & $\begin{array}{l}-p(N-x)+x(1-p) \\
* \sqrt{\frac{(N-1) ! p^{x-1}(1-p)^{N-x-1}}{x !(N-x) !}}\end{array}$ & $\frac{\mu-x}{\mu} \sqrt{\frac{\mathrm{e}^{-\mu} \mu^{x+1}}{x !}}$ \\
\hline
\end{tabular}

Table 4. Zero and first order polynomials of the following functions: Hahn $\tilde{h}_{n}^{a, b}(x ; N)$, Meixner $\tilde{m}_{n}^{\beta, \mu}(x)$ y Racah.

\begin{tabular}{ccc}
\hline$\tilde{h}_{n}^{a, b}(x ; N)$ & $\tilde{m}_{n}^{\beta, \mu}(x)$ \\
\hline$\tilde{p}_{0}(x)$ & $\sqrt{\frac{(a+1)_{b}(a+b+1)}{(N-a)_{b+1}}}$ & $\sqrt{\frac{\mu^{x}(\beta+x-1) !}{x !(\beta-1) !(1-\mu)^{\beta}}}$ \\
$\tilde{p}_{1}(x)$ & $* \sqrt{\frac{(a+b+2) x-(b+1)(N-1)}{(a+1)(b+1)(n-1)(N+1+b+1)}}$ & $\left(\beta+x-\frac{x}{\mu}\right) \sqrt{\frac{\mu^{x}(\beta+x-1) !}{x !(\beta-1) !} \frac{\mu(1-\mu)^{\beta}}{\beta}}$
\end{tabular}

$U_{n-2}^{\alpha, \beta}(s, a, b)$ are the initial values of Zero and first order polynomials shown in “Table 5".

Below, the graphs obtained from the calculation of the first 5 polynomials of the six families of discrete orthogonal moments are shown in Figure 1.

\section{RGB Color Images Reconstruction}

One of the different applications of the moments' functions with orthogonal base is the reconstruction of color images $f(x, y, c)$, which allows to determine the number of descriptors that can characterize the color image.

It is possible to reconstruct an image from its moments using the respective inverse transformation. The reconstruction of an image $f(x, y)$ is given by [4]:

$$
\tilde{f}(x, y)=\sum_{x=1}^{m} \sum_{y=1}^{n} \varphi_{m, n} P_{n}(x) P_{m}(y),
$$

where $P_{n}(x)$ and $P_{m}(x)$ are polynomial basis functions and $\varphi_{m, n}$ are the moments of the image to be reconstructed.

The reconstruction of a color image $f(x, y, c)$ is given by:

$$
\tilde{f}_{c}(x, y)=\sum_{x=1}^{m} \sum_{y=1}^{n} \varphi_{n, m}^{c} P_{n}(x) P_{m}(y),
$$

where $P_{n}(x)$ y $P_{m}(y)$ are polynomial basis functions and $\varphi_{n, m}^{c}$ are the moments of the image to be reconstructed in its three RGB channels. 
Table 5. Values to obtain the Racah polynomials $\tilde{u}_{n}^{\alpha, \beta}(s, a, b)$.

$$
\begin{aligned}
& \tilde{p}_{0}(x) \\
& \sqrt{\frac{(2 s+1)(p(s))}{d_{0}^{2}}} \\
& \frac{p_{1}(s)-p(s-1)}{x\left(s+\frac{1}{2}\right)-x\left(s-\frac{1}{2}\right)} \frac{1}{p(s)} * \sqrt{\frac{\mathrm{e}^{-\mu} \mu^{x+1}}{x !}} \\
& A_{n} \\
& \frac{n(\alpha+\beta+n)}{(\alpha+\beta+2 n-1)(\alpha+\beta+2 n)} \\
& x-\frac{a^{2}+b^{2}+(a-\beta)^{2}(b+\alpha)^{2}-2(\alpha+\beta+2 n-2)(\alpha+\beta-2)}{4} \\
& +\frac{(\alpha+\beta+2 n-2)-(\alpha+\beta+2 n)}{8}-\frac{\left(\beta^{2}-\alpha^{2}\right)\left[\left(b+\frac{\alpha}{2}\right)^{2}-(a-\beta / 2)^{2}\right]}{2(\alpha+\beta+2 n-2)(\alpha+\beta+2 n)} \\
& -\frac{(\alpha+n-1)(\beta+n-1)}{(\alpha+\beta+2 n-2)(\alpha+\beta+2 n)} *\left[\left(a+b+\frac{\alpha-\beta}{2}\right)^{2}\left(n-1+\frac{\alpha+\beta}{2}\right)^{2}\right] \\
& *\left[\left(b+a+\frac{\alpha+\beta}{2}\right)^{2}\left(n-1+\frac{\alpha+\beta}{2}\right)^{2}\right] \\
& d_{n} \quad \frac{\Gamma(\alpha+n+1) \Gamma(\beta+n+1) \Gamma(b-a+\alpha+\beta+n+1) \Gamma(a+b+\alpha+n+1)}{(\alpha+\beta+2 n+1) n !(b-a-n-1) \Gamma(\alpha+\beta+n+1) \Gamma(a+b-\beta-n)} \\
& \frac{\Gamma(\alpha+s+n+1) \Gamma(s-a+\beta+n+1) \Gamma(N+\alpha-s) \Gamma(N+\alpha+s+n+1)}{\Gamma(a-\beta+s+1) \Gamma(s-a+1) \Gamma(N-s-n) \Gamma(N+s+1)}
\end{aligned}
$$

\section{RGB Image Reconstruction Error}

To evaluate the efficiency of the moments, the reconstruction metric based on the normalized image reconstruction error (NIRE) is used, which is defined as the normalized square error between the input image $f_{c}(x, y)$ and the reconstructed image $\tilde{f}_{c}(x, y, c)$, expressed by [4]:

$$
\operatorname{NIRE}_{c}=\frac{\sum_{y=1}^{N} \sum_{x=1}^{M}\left[f_{c}(x, y)-\tilde{f}_{c}(x, y)\right]^{2}}{\sum_{y=1}^{N} \sum_{x=1}^{M} f_{c}^{2}(x, y)}
$$

Therefore, the measurement for the reconstruction error of a color image that takes into account the three channels is given by:

$$
\text { MeanNIRE }=\frac{\mathrm{NIRE}_{R}+\mathrm{NIRE}_{G}+\mathrm{NIRE}_{B}}{3}
$$

\section{Experimental Results}

Next are the results obtained with the proposed algorithm for video encryption and recovery of the following three grayscale test images with dimensions of $512 \times$ 


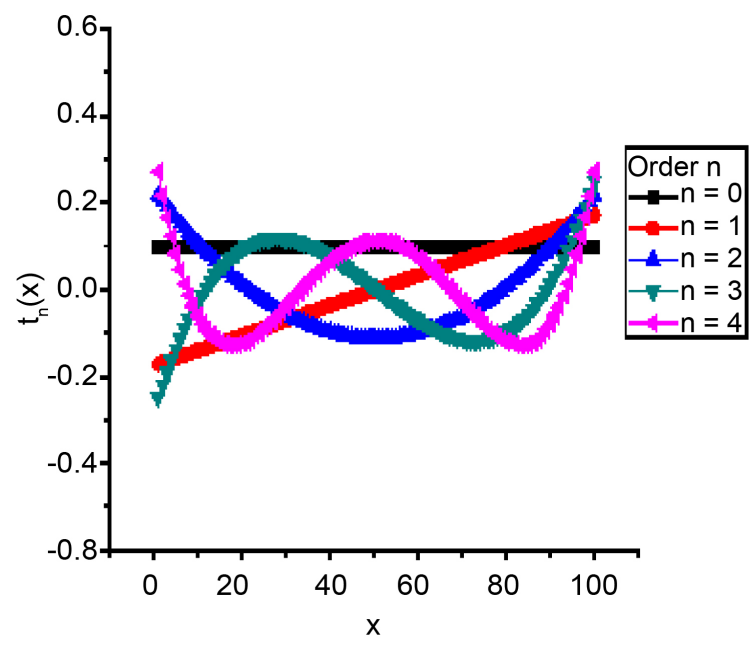

(a)

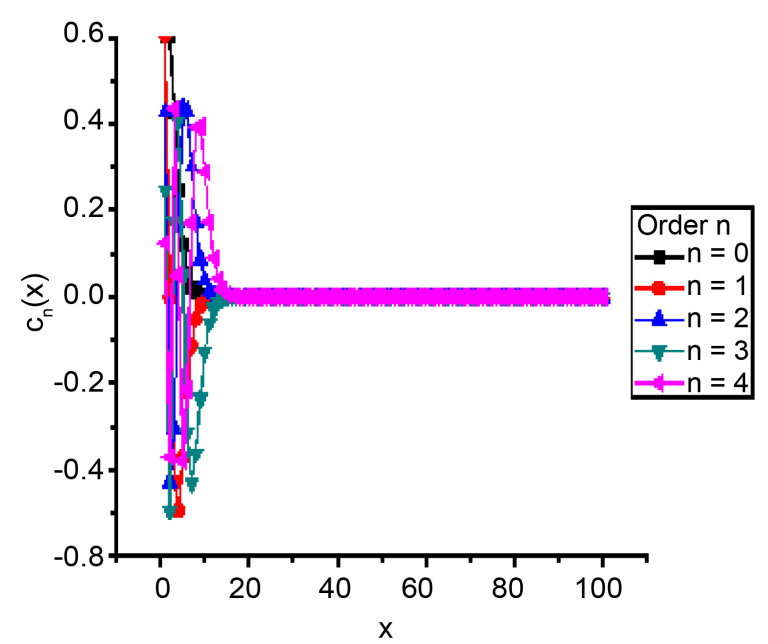

(c)

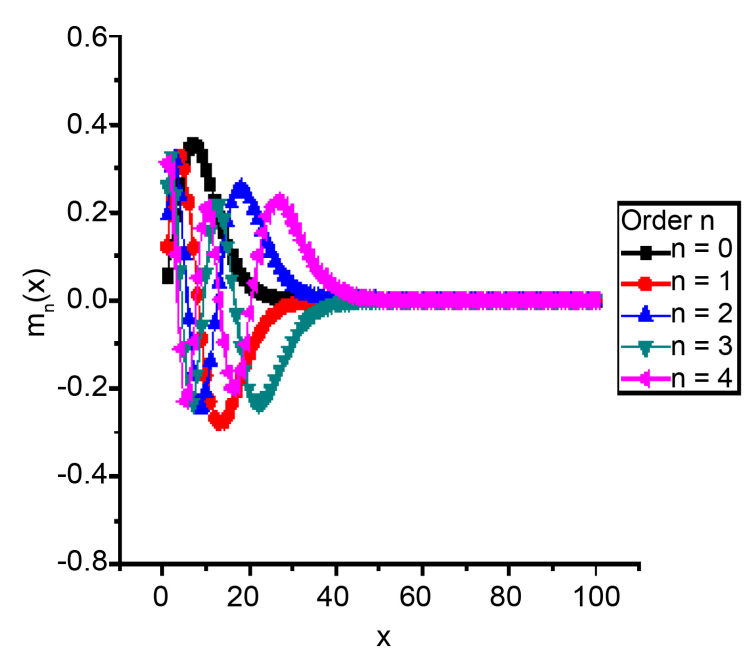

(e)

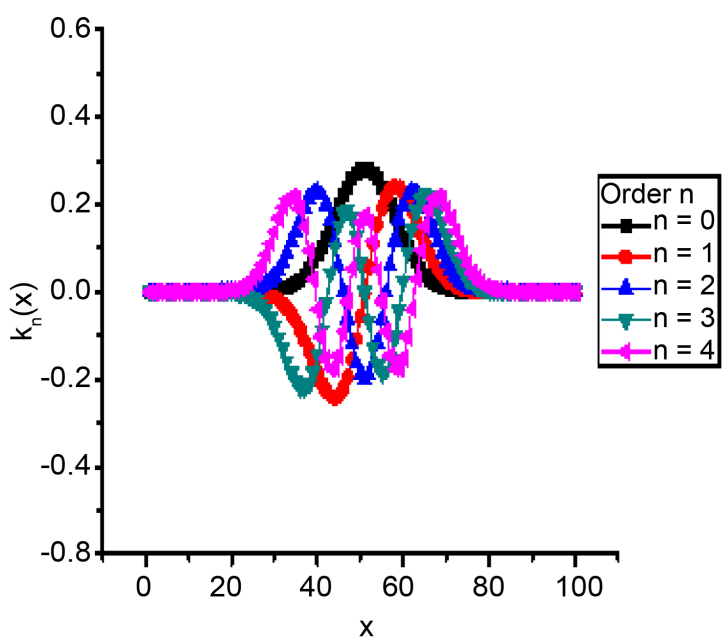

(b)

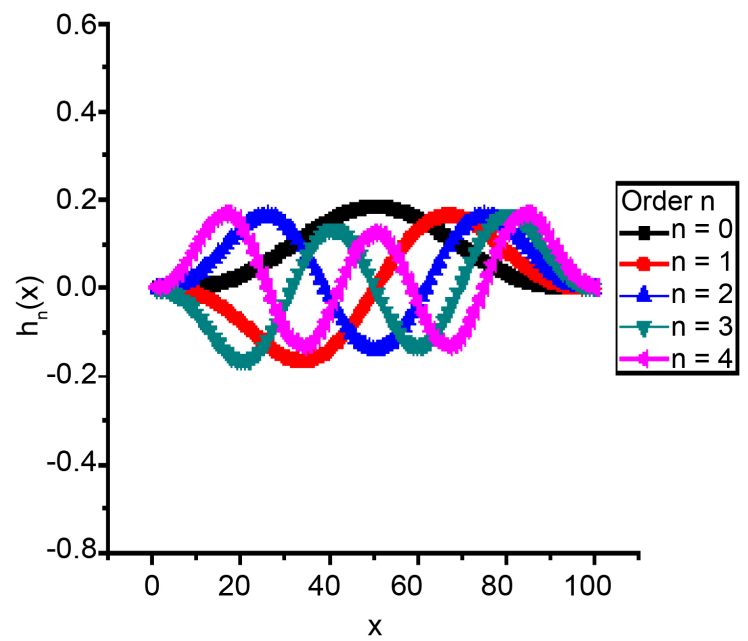

(d)

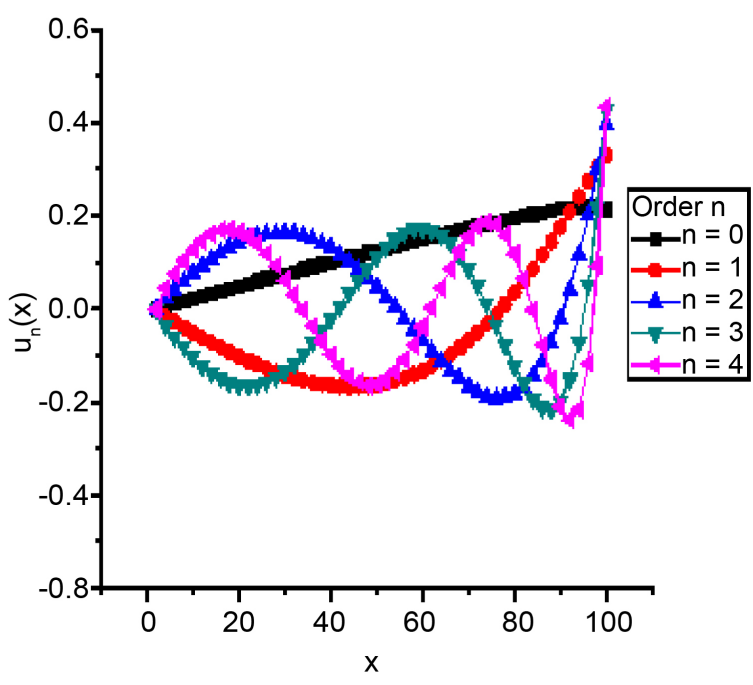

(f)

Figure 1. The first ten polynomials $\mathrm{n}=0,1, \ldots, 4$. (a) Tchebichef polynomials, (b) Krawtchouk polynomials, (c) Charlier polynomials, (d) Hahn polynimials, (e) Meixner polynomials and (f) Racah polynomials. 
512 pixels presented in Figure 2.

We have reconstructed these test images shown in Figure 2 with the moments of the different families of classical polynomials with different orders of reconstruction. The results obtained with the reconstruction are shown in "Tables 6-8”.

Image 1

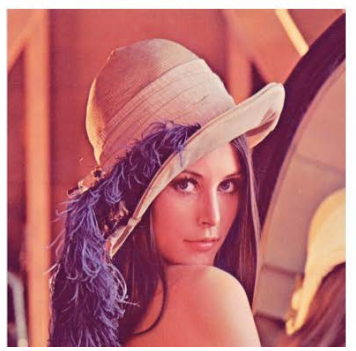

Image 2

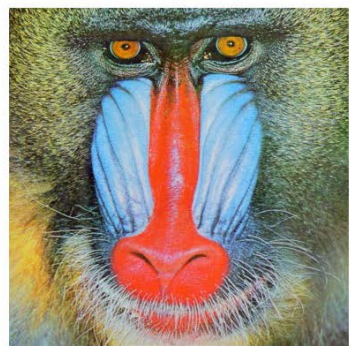

Image 3

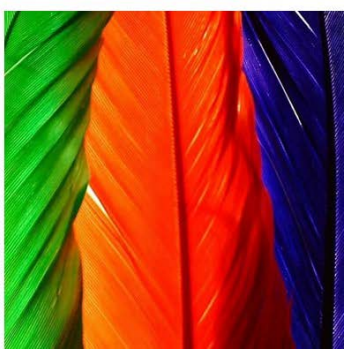

Figure 2. Grayscale image of $512 \times 512$ used as images for video encryption (a) "lena.jpg", (b) "mandril.jpg" and (c) "plumas.jpg".

Table 6. Comparison of some pictures of the reconstruction orders of image 1, "lena" with each one of the moments sets mentioned.

\begin{tabular}{|c|c|c|c|c|}
\hline \multirow{2}{*}{ Moments } & \multicolumn{4}{|c|}{ Reconstruction orders } \\
\hline & 10 & 25 & 50 & 100 \\
\hline Tchebichef & & & & \\
\hline Krawtchouk & & & & \\
\hline Charlier & & & & \\
\hline Hahn & & & & \\
\hline Meixner & & & & \\
\hline Racah & & & & \\
\hline
\end{tabular}


Table 7. Comparison of some pictures of the reconstruction orders of image 2, "Mandril" with each one of the moments sets mentioned.

\begin{tabular}{|c|c|c|c|c|}
\hline \multirow{2}{*}{ Moments } & \multicolumn{4}{|c|}{ Reconstruction orders } \\
\hline & 10 & 25 & 50 & 100 \\
\hline Tchebichef & & & & \\
\hline Krawtchouk & & & & \\
\hline Charlier & & & & \\
\hline Hahn & & & & \\
\hline Meixner & & & & \\
\hline Racah & & & & \\
\hline
\end{tabular}

The NIRE graphics of the reconstruction comparison of the three images with the moments sets are shown in Figure 3.

\section{Conclusions}

According to the value distributions of each set of orthogonal polynomials as shown in Figure 1, it can be observed that the distribution of some polynomials as Tchebichef and Hahn are more uniform in comparison to Krawtchouk, Meixner, Charlier and Racah polynomials. Therefore, better results would be 
Table 8. Comparison of some pictures of the reconstruction orders of image 3, "Plumas" with each one of the moments sets mentioned.

\begin{tabular}{|c|c|c|c|c|}
\hline \multirow{2}{*}{ Moments } & \multicolumn{4}{|c|}{ reconstruction orders } \\
\hline & 10 & 25 & 50 & 100 \\
\hline Tchebichef & & & & \\
\hline Krawtchouk & & & & \\
\hline Charlier & & & & \\
\hline Hahn & & & & \\
\hline Meixner & & & & \\
\hline Racah & & & & \\
\hline
\end{tabular}

expected with these polynomials.

Rectangular moments were used for the reconstruction of an image, with which it was possible to examine the performance of the different moments used for the representation of the information, as well as its convergence through the number of moments necessary for the reconstruction of the images.

As can be seen in the results obtained shown in Tables 6-8. The Tchebichef, Hahn and Racah polynomials present a better capacity of description, since they allow to recover the image with a smaller number of reconstruction orders. In 


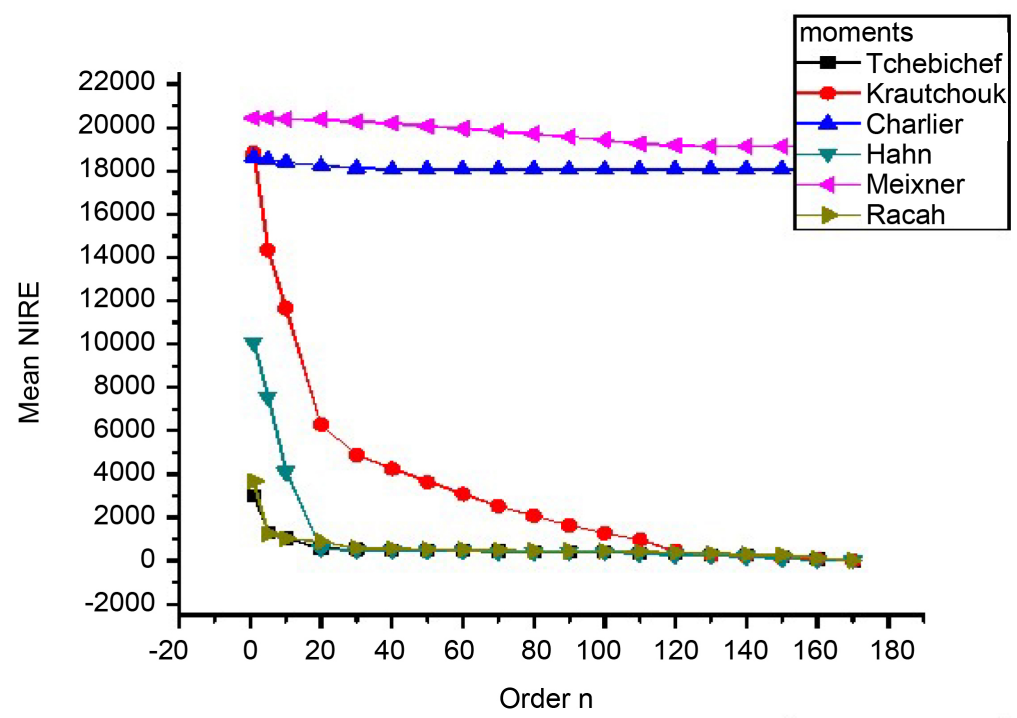

(a)

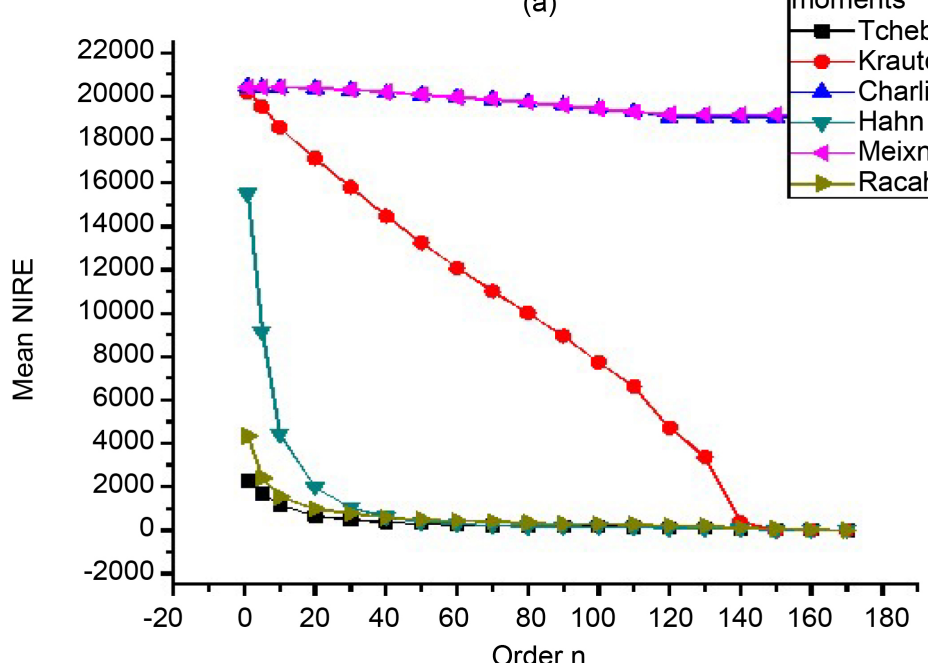

(b)

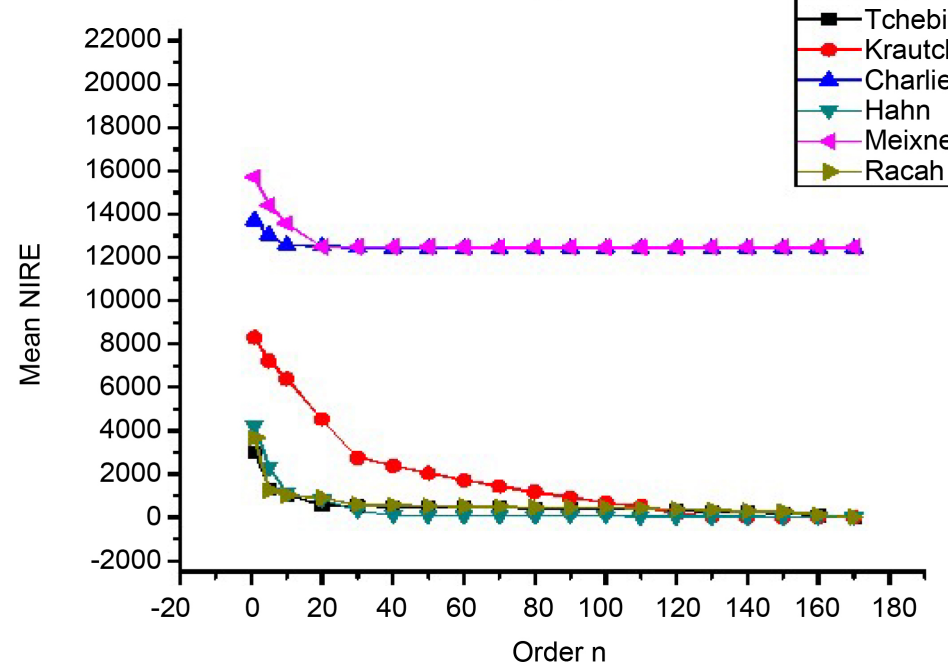

(c)

Figure 3. Graphics of the reconstruction comparison of error for images (a) "lena.jpg", (b) "mandril.jpg" and (c) "plumas.jpg". 
this case with more than 50 orders it can recover almost the entire image.

\section{Acknowledgements}

J. S. Rivera-López thanks CONACyT for the scholarship with number 423649.

\section{References}

[1] Suk, T. and Flusser, J. (2009) Affine Moment Invariants of Color Images. In: Proceedings of CAIP 2009, LNCS5702, 334-341.

https://doi.org/10.1007/978-3-642-03767-2_41

[2] Chen, B.J., Shu, H.Z., Zhang, H., Chen, G., Toumoulin, C., Dillenseger, J.L. and Luo, L.M. (2012) Quaternion Zernike Moments and Their Invariants for Color Image Analysis and Object Recognition. In Signal Processing, 92, 308-318.

https://doi.org/10.1016/j.sigpro.2011.07.018

[3] Chen, B., Shu, H., Zhang, H., Chen, G. and Luo, L. (2010) Color Image Analysis by Quaternion Zernike Moments. 2010 20th International Conference on Pattern Recognition, Istanbul, 23-26 Aug. 2010, 625-628.

https://doi.org/10.1109/ICPR.2010.158

[4] Camacho-Bello, C., Padilla-Vivanco, A., Toxqui-Quitl, C. and Báez-Rojas, J.J. (2016) Reconstruction of Color Biomedical Images by Means of Quaternion Generic Jacobi-Fourier Moments in the Framework of Polar Pixels. Journal of Medical Imaging, 3, 014004-014004. https://doi.org/10.1117/1.JMI.3.1.014004

[5] Mukundan, R., Ong, S.H. and Lee, P.A. (2001) Image Analysis by Tchebichef Moments. IEEE Transactions on image Processing, 10, 1357-1364.

https://doi.org/10.1109/83.941859

[6] Yap, P.T., Paramesran, R. and Ong, S.H. (2003) Image Analysis by Krawtchouk Moments. IEEE Transactions on Image Processing, 12, 1367-1377. https://doi.org/10.1109/TIP.2003.818019

[7] Zhou, J., Shu, H., Zhu, H., Toumoulin, C. and Luo, L. (2005) Image Analysis by Discrete Orthogonal Hahn Moments. Image Analysis and Recognition, 3656, 524-531. https://doi.org/10.1007/11559573_65

[8] Sayyouri, M., Hmimid, A. and Qjidaa, H. (2015) A Fast Computation of Novel Set of Meixner Invariant Moments for Image Analysis. Circuits, Systems, and Signal Processing, 34, 875-900. https://doi.org/10.1007/s00034-014-9881-7

[9] Zhang, X. and Liao, S. (2015) Color Image Reconstruction from Charlier Moments. Journal of Theoretical and Applied Computer Science, 9, 3-13.

[10] Wu, Y. and Liao, S. (2015) Color Image Analysis via Racah Moments. Journal of Theoretical and Applied Computer Science, 9, 8-18.

[11] Wu, Y. and Liao, S. (2014) Chinese Characters Recognition via Racah Moments. In Audio, Language and Image Processing (ICALIP), 2014 International Conference on, IEEE, 7-9 July 2014, 691-694. https://doi.org/10.1109/ICALIP.2014.7009883

[12] Zhu, H., Liu, M., Shu, H., Zhang, H. and Luo, L. (2010) General Form for Obtaining Discrete Orthogonal Moments. IET Image Processing, 4, 335-352. https://doi.org/10.1049/iet-ipr.2009.0195 